\title{
Clinical Predictors of Transient versus Persistent Neonatal Hyperinsulinism
}

\author{
Nicole Falzone ${ }^{a}$ Jennifer Harrington ${ }^{a, b}$ \\ ${ }^{a}$ Faculty of Medicine, University of Toronto, Toronto, ON, Canada; ${ }^{b}$ Department of Pediatrics, Division of \\ Endocrinology, The Hospital for Sick Children, University of Toronto, Toronto, ON, Canada
}

\section{Keywords}

Hyperinsulinism · Hypoglycemia · Length of stay · Genetic testing

\begin{abstract}
Introduction: Hyperinsulinism (HI), the most common neonatal cause of persistent hypoglycemia, can be associated with prolonged hospitalizations and risk for long-term neurological sequelae. Rapid identification of transient versus persistent forms of $\mathrm{HI}$ is crucial to optimize management. Objectives: The aims of the study were to assess the ability of clinical and biochemical features at presentation to predict transient versus persistent $\mathrm{HI}$, and to evaluate differences in hospital outcomes. Methods: This study is a retrospective review of 79 infants with $\mathrm{HI}$ admitted to the Hospital for Sick Children, Toronto, from 2012 to 2017. Patients were classified into 3 groups: transient and the 2 persistent forms, diazoxide responsive and diazoxide unresponsive (DU). $\boldsymbol{R} \boldsymbol{e}$ sults: Infants with birth weight >90th percentile had an 8 -fold increased risk of having a persistent form of $\mathrm{HI}$ (OR 8.8, $95 \% \mathrm{Cl} 2.5-30)$ and a 21 -fold increased risk of having a DU form of HI (OR 21.1, 95\% Cl 4.9-91.8). The majority of children with transient $\mathrm{HI}$ and a birth weight $>90$ th percentile were born to mothers with gestational diabetes. There were no other useful clinical or biochemical presenting features that differentiated the groups. There were significant differences in outcome measures, with the DU children more likely to
\end{abstract}

require gastrostomy tube insertion and have an extended length of hospital admission. Conclusion: A higher birth weight in the absence of maternal gestational diabetes is highly associated with a persistent form of HI. Given the marked difference in clinical outcomes between groups, expedited genetic testing should be considered in infants with this presentation to inform clinical management.

(c) 2020 The Author(s)

Published by S. Karger AG, Basel

\section{Introduction}

Hyperinsulinism (HI) is a condition of unregulated and inappropriate insulin secretion from pancreatic $\beta$-cells, which results in hypoglycemia and is the most common cause of persistent hypoglycemia in neonates [1]. Infants with $\mathrm{HI}$ are at increased risk of developing irreversible cerebral injury and long-term neurological deficits, including developmental delay and cerebral palsy [2, $3]$. HI typically presents in neonates, most often in the first 2-4 h of life; however, later presentations are also observed [4]. Blood glucose testing is indicated in at-risk (small for gestational age [SGA], large for gestational age [LGA], infants of diabetic mothers, or preterm infants) or unwell neonates, and is the first biochemical indicator of HI [5]. Late-presenting infants often present to hospital emergency departments with symptoms of hypoglycemia, prompting appropriate investigations and treatment.

\section{karger@karger.com www.karger.com/hrp

\section{(C) 2020 The Author(s)}

Published by S. Karger AG, Basel

This is an Open Access article licensed under the Creative Commons Attribution-NonCommercial-4.0 International License (CC BY-NC) (http://www.karger.com/Services/OpenAccessLicense), applicable to the online version of the article only. Usage and distribution for commercial purposes requires written permission.
Jennifer Harrington

Division of Endocrinology, The Hospital for Sick Children

555 University Avenue

Toronto ON M5G1X8 (Canada)

jennifer.harrington@ sickkids.ca 
HI is a broad classification that encompasses a heterogeneous group of disorders, including both transient and congenital forms of the disease. Transient HI often occurs in the newborn period, with intrauterine growth restriction, fetal distress, and maternal gestational diabetes commonly cited as risk factors [4]. The disease course is usually self-limiting and resolves in the first few weeks to months of life. Initial management of $\mathrm{HI}$ is targeted at maintaining normoglycemia. Glycemic control is achieved through supportive management including providing increased glucose infusions (either orally or intravenously) and eventual intravenous glucagon infusions. Long-term therapy consists of diazoxide, as firstline treatment for HI, or somatostatin analogs in case of diazoxide unresponsiveness [6].

Congenital $\mathrm{HI}$ is a result of a genetic mutation in the pancreatic $\beta$-cell that increases insulin secretion. In the pancreatic $\beta$-cell, glucose-dependent insulin secretion occurs when glucose enters the $\beta$-cell via a glucose transporter protein. Through a series of signaling pathways, the ATP:ADP ratio is increased, causing closure of the $[\mathrm{K}]^{+}$ ATP channel and subsequent depolarization of the cell membrane. Depolarization causes an influx of $[\mathrm{Ca}]^{2+}$ ions, resulting in exocytosis of insulin-filled granules [7-9]. Usually, mutations along this pathway before the $[\mathrm{K}]^{+}$ ATP channel cause a form of HI that responds to diazoxide, which works to keep the $[\mathrm{K}]^{+}$ATP channel open and prevent the efflux of insulin [10]. In contrast, mutations affecting the $[\mathrm{K}]^{+}$ATP channel itself and causing it to remain closed ( $A B C C 8 / K C N J 11$ mutations) are generally not susceptible to diazoxide, requiring alternative therapeutic modalities including octreotide or surgical management. These persistent forms of HI can, therefore, be further categorized into diazoxide responsive (DR) and diazoxide unresponsive (DU).

Recent literature has demonstrated an association between hyperinsulinemic hypoglycemia and increased length and cost of hospital stay [11]. The aims of this study were to assess whether there are differences in both clinical presentation and outcome variables between patients with the 3 different forms of $\mathrm{HI}$ (transient and the 2 persistent forms, which for the purposes of this study are labeled DR and DU) that could be used to help guide clinicians to streamline treatment approaches.

\section{Materials and Methods}

This was a retrospective chart review of infants who were seen by the Endocrinology Service at the Hospital for Sick Children with a diagnosis of HI between June 2012 and March 2017. Sub- jects were identified using divisional diagnostic databases. Children presenting with hypoglycemia secondary to a cause other than $\mathrm{HI}$, presenting with $\mathrm{HI}$ outside the neonatal period (1 month of life), and consulted exclusively at peripheral hospitals were excluded from the study. Data were extracted from electronic medical records into the REDCap database.

Data were collected for birth history, clinical and biochemical features at the time of diagnosis, genetic testing, management of HI during initial hospitalization, documented feeding issues (defined as vomiting from presumed reflux, inability to take feeds orally, swallowing problems, or other documented problems including not tolerating oral feeds and feeding aversion), duration of stay, and discharge medications. While there is variation based on clinician practice, genetic testing is offered to families if $\mathrm{HI}$ persists beyond 1 year or in DU subjects. Genetic testing was performed using the next-generation sequencing panel which included the following genes: KCNJ11, ABCC8, $H A D H$, HNF4A, GLUD1, GCK, SLC16A1, TRMT10A, HNF1A, and INSR. Birth weight $Z$ scores for gestational age were calculated using the Canadian Pediatric Endocrine Group preterm calculator based upon published norms [12, 13]. Following data collection, subjects were divided into 3 groups for statistical analysis: transient, persistent DR, and persistent DU. For the purposes of this study, transient HI was defined as subjects diagnosed with HI (an inappropriately elevated or measurable insulin concentration at the time of a serum blood glucose $<2.7 \mathrm{mmol} / \mathrm{L}$ in children with persistent hypoglycemia requiring a glucose infusion of $>8 \mathrm{mg} / \mathrm{kg} / \mathrm{min}$ ) that resolved by 4 months of age. The cutoff of 4 months was determined based on evidence that transient forms of HI should show resolution of symptoms by this age [14, 15]. While the schedule of weaning the diazoxide was based upon clinical practice of the individual physician, in general the dose was reduced by $25 \%$ every week, provided all home blood glucose monitoring numbers were $>3.5 \mathrm{mmol} / \mathrm{L}$. The resolution of $\mathrm{HI}$ was determined by the subject's ability to be off therapy and pass a 6-h fasting study with no residual evidence of hyperinsulinemia. In contrast, persistent $\mathrm{HI}$ was defined as the need for ongoing therapy after 4 months of age. The term persistent rather than congenital HI was used, given not all infants had genetic testing. Diazoxide responsiveness was defined as maintenance of normoglycemia (blood glucose $>3.5 \mathrm{mmol} / \mathrm{L}$ ) on diazoxide therapy alone up to a maximum dose of $15 \mathrm{mg} / \mathrm{kg} /$ day and passing of a fasting test for a minimum of $6 \mathrm{~h}$ prior to discharge home. Infants of mothers with gestational onset diabetes and those with perinatal stress were included if referred to our center as this does not preclude a diagnosis of HI. Children with insufficient follow-up and those with an adverse reaction to the diazoxide treatment (and thus responsiveness to diazoxide could not be determined) were excluded from the study analysis.

Data were analyzed by SPSS 25.0 for Windows (SPSS Inc., Chicago, IL, USA), and descriptive statistics were generated for the cohort. Differences in categorical variables by diagnostic group were assessed using $\chi^{2}$ analysis. Continuous variables were assessed using independent sample $t$ tests and one-way ANOVA with Pearson's correlation for the post hoc analysis. Variables with a $p$ value $<0.1$ in the initial analysis were included in a logistic regression to assess predictors of the diagnostic group. Contributors to length of stay were analyzed by a multiregression model while controlling for group (transient, DR, and DU). 
Table 1. Genetic testing results

\begin{tabular}{llll}
\hline Genetic testing outcome & $\begin{array}{l}\mathrm{T} \\
(n=46)\end{array}$ & $\begin{array}{l}\mathrm{DR} \\
(n=20)\end{array}$ & $\begin{array}{l}\mathrm{DU} \\
(n=13)\end{array}$ \\
\hline $\begin{array}{l}\text { Number (\%) of patients for whom HI-related genetic testing was sent } \\
\text { Genetic mutation results (number of subjects) }\end{array}$ & $11(24)$ & $15(75)$ & $11(85)$ \\
ABCC8 heterozygous mutation & 0 & 0 & 3 \\
ABCC8 homozygous/compound heterozygous mutation & 0 & 0 & 6 \\
GLUD1 mutation & 0 & 3 & 0 \\
HNF4A mutation & 0 & 3 & 0 \\
HADH mutation & 0 & 1 & 0 \\
Turner's syndrome & 1 & 0 & 0 \\
No mutation found & 10 & 8 & 2 \\
\hline
\end{tabular}

T, transient; DR, diazoxide responsive; DU, diazoxide unresponsive. ${ }^{\mathrm{a}}$ Of the DU group, 3 had focal disease, all of which were treated surgically. Of the 10 patients with diffuse disease, 3 were treated with a near-total pancreatectomy and 7 with medical treatment. ${ }^{b}$ All patients in the DU cohort were offered genetic testing; however, 2 families declined to have genetic testing sent.

Table 2. Cohort characteristics

\begin{tabular}{|c|c|c|c|c|c|}
\hline Characteristic & $\begin{array}{l}\text { Total cohort } \\
(n=79)\end{array}$ & $\begin{array}{l}\mathrm{T} \\
(n=46)\end{array}$ & $\begin{array}{l}\mathrm{DR} \\
(n=20)\end{array}$ & $\begin{array}{l}\mathrm{DU} \\
(n=13)\end{array}$ & $p$ value \\
\hline Sex, male, \% & 59.4 & 78.3 & 35.0 & 30.8 & 0.001 \\
\hline Gestational age, weeks & $37.4 \pm 2.6$ & $37.2 \pm 2.5$ & $37.9 \pm 2.3$ & $37.6 \pm 3.5$ & 0.60 \\
\hline Prematurity, \% & 32.9 & 37.0 & 25.0 & 30.8 & 0.63 \\
\hline Birth weight ( $Z$ score) & $-0.3 \pm 1.7$ & $-0.8 \pm 1.5$ & $-0.1 \pm 1.7$ & $1.4 \pm 1.5$ & $<0.001^{* *, * * *}$ \\
\hline LGA, \%a & 24.1 & 8.7 & 25.0 & 76.9 & $<0.001^{*, * *}$ \\
\hline SGA, $\%^{\mathrm{a}}$ & 36.7 & 47.8 & 25 & 15.4 & $0.04^{* *}$ \\
\hline C-section, \% & 61.5 & 67.4 & 52.6 & 53.8 & 0.44 \\
\hline Fetal distress, $\%$ & 48.7 & 54.3 & 42.1 & 38.5 & 0.48 \\
\hline Gestational maternal diabetes, $\%$ & 10.1 & 10.9 & 10.0 & 7.7 & 0.95 \\
\hline Presence of SGA, fetal distress, or maternal diabetes, \% & 69.6 & 73.9 & 65.0 & 53.8 & 0.36 \\
\hline Patients with first documented hypoglycemic event in first $24 \mathrm{~h}$ of life, $\%$ & 73.4 & 73.9 & 70.0 & 76.9 & 0.90 \\
\hline
\end{tabular}

Data are represented as mean \pm standard deviation or \%. LGA, large for gestational age; SGA, small for gestational age; T, transient; DR, diazoxide responsive; DU, diazoxide unresponsive. $p<0.05 .{ }^{*}$ T versus DR. ${ }^{* *}$ T versus DU. ${ }^{* *}$ DR, versus DU, using Bonferroni post hoc analysis. ${ }^{a}$ LGA defined by a birth weight $Z$ score of $>1.2$ and SGA by a birth weight $Z$ score of $<-1.2$.

This study was approved by the Research and Ethics Board at the Hospital for Sick Children, Toronto. Approval to waive the need for informed consent was given by the Ethics Committees, given the retrospective nature of the chart review.

\section{Results}

\section{Cohort Characteristics}

A total of 137 patient charts were reviewed for inclusion into the study. Forty-one were excluded because the etiology of hypoglycemia was not due to HI or they had been only seen at a community hospital; a further 14 chil- dren were excluded because their HI presented outside the neonatal period and 3 infants were excluded as they developed early adverse effects to diazoxide and hence could not be categorized as responsive or unresponsive to diazoxide. The cohort of infants diagnosed with HI ( $n=$ $79,59.4 \%$ male) was divided into 3 groups: transient ( $n=$ $46,78.3 \%$ male $)$ and the 2 persistent subtypes: $\operatorname{DR}(n=20$, $35 \%$ male) and DU ( $n=13,30.8 \%$ male). Genetic testing for mutations associated with $\mathrm{HI}$ occurred in $75 \%$ of the DU and $85 \%$ of the DR groups compared to $24 \%$ of the transient group. Genetic causes of $\mathrm{HI}$ in this cohort are summarized in Table 1. 
Table 3. Clinical predictors: critical sample biochemistry and age of presentation

\begin{tabular}{lccccc}
\hline & $\begin{array}{l}\text { Total cohort } \\
(n=79)\end{array}$ & $\begin{array}{l}\mathrm{T} \\
(n=46)\end{array}$ & $\begin{array}{l}\mathrm{DR} \\
(n=20)\end{array}$ & $\begin{array}{l}\mathrm{DU} \\
(n=13)\end{array}$ & $p$ value \\
\hline Glucose, $\mathrm{mmol} / \mathrm{L}$ & $2.1 \pm 0.6$ & $2.2 \pm 0.6$ & $2.1 \pm 0.5$ & $1.8 \pm 0.6$ & 0.12 \\
Insulin, $\mathrm{pmol} / \mathrm{L}$ & $110.4 \pm 154$ & $89.8 \pm 127$ & $116.9 \pm 132$ & $177 \pm 262$ & 0.22 \\
Serum ketones, $\mathrm{mmol} / \mathrm{L}$ & $0.07 \pm 0.07$ & $0.07 \pm 0.05$ & $0.06 \pm 0.05$ & $0.07 \pm 0.07$ & 0.91 \\
Free fatty acid, $\mathrm{mmol} / \mathrm{L}$ & $0.16 \pm 0.14$ & $0.16 \pm 0.15$ & $0.16 \pm 0.10$ & $0.14 \pm 0.15$ & 0.91 \\
Cortisol, nmol/L & $311 \pm 290$ & $263 \pm 276$ & $302 \pm 169$ & $536 \pm 427$ & $0.03^{*}$ \\
Growth hormone, $\mu \mathrm{g} / \mathrm{L}$ & $13.9 \pm 9.7$ & $14.2 \pm 9.7$ & $14.4 \pm 12.6$ & $10.9 \pm 4.8$ & 0.59 \\
\hline
\end{tabular}

Data are represented as mean \pm standard deviation. T, transient; DR, diazoxide responsive; DU, diazoxide unresponsive; HI, hyperinsulinism. $p<0.05$. ${ }^{*}$ T versus DU, using Bonferroni post hoc analysis.

Table 4. Hospital course of patients by HI subgroup

\begin{tabular}{|c|c|c|c|c|c|}
\hline & $\begin{array}{l}\text { Total cohort } \\
(n=79)\end{array}$ & $\begin{array}{l}\mathrm{T} \\
(n=46)\end{array}$ & $\begin{array}{l}\mathrm{DR} \\
(n=20)\end{array}$ & $\begin{array}{l}\mathrm{DU} \\
(n=13)\end{array}$ & $p$ value \\
\hline Maximum GIR, mg/kg/min & $14.5[7.3-26.4]$ & $14.1[7.3-19.7]$ & $14.7[11.5-26.4]$ & $15.7[9-24.3]$ & 0.29 \\
\hline Glucagon duration, days & $19[2-123]$ & $14[6-29]$ & $15[2-60]$ & $42[6-123]$ & $<0.001^{* *, * * *}$ \\
\hline Percentage of patients given feed fortification & 87.5 & 83.3 & 94.1 & 92.3 & 0.44 \\
\hline Documented feeding problem, $\%$ & 61.8 & 56.8 & 63.2 & 76.9 & 0.42 \\
\hline Age diazoxide started, days & $17 \pm 10$ & $20 \pm 11^{\mathrm{a}}$ & $18 \pm 9$ & $11 \pm 5$ & 0.28 \\
\hline Maximal diazoxide dose, $\mathrm{mg} / \mathrm{kg} / \mathrm{day}$ & $11.0 \pm 3.6$ & $9.5 \pm 2.2$ & $11.0 \pm 3.7$ & $15.3 \pm 3.3$ & $<0.001^{* *, * * *}$ \\
\hline Duration of diazoxide, days & $1,162 \pm 5,405$ & $81 \pm 40$ & $626 \pm 557^{b}$ & $31 \pm 20$ & $0.014^{*}$ \\
\hline Length of hospital admission, days & $53.8 \pm 46.8$ & $39.6 \pm 26.3$ & $50.7 \pm 26.4$ & $106.8 \pm 79.9$ & $<0.001^{* *, * * *}$ \\
\hline $\begin{array}{l}\text { Length of hospital admission, days (excluding } \\
\text { premature infants) }\end{array}$ & $47.8 \pm 44.6$ & $32.5 \pm 16.4$ & $45.1 \pm 26.2$ & $99.6 \pm 90.9$ & $<0.001^{* *, * * *}$ \\
\hline
\end{tabular}

Data are represented as mean [range], mean \pm standard deviation, or \%. T, transient; DR, diazoxide responsive; DU, diazoxide unresponsive; HI, hyperinsulinism. ${ }^{*}$ T versus DR. ${ }^{* *}$ T versus DU. ${ }^{* * *}$ DR versus DU, using Bonferroni post hoc analysis. ${ }^{\text {a }}$ Eight of 46 transient cohort not commenced on diazoxide and were treated with glucagon alone. ${ }^{\mathrm{b}}$ Ten of the $20 \mathrm{DR}$ subjects still on diazoxide treatment at the time of analysis.

\section{Presenting Clinical Predictors for Type of HI}

The cohort clinical characteristics are presented in $\mathrm{Ta}$ ble 2. Age of first hypoglycemic event, gestational age, and prematurity rates were not statistically different between groups.

Diazoxide unresponsiveness was associated with increased birth weight $Z$ score. Infants with birth weight $Z$ scores $>1.2$ (equivalent to $>90$ th percentile or LGA) had an 8 -fold increased risk of having a persistent form of $\mathrm{HI}$ (OR 8.8, 95\% CI 2.5-30) and a 21 -fold increased risk of having a DU form of HI (OR 21.1, 95\% CI 4.9-91.8). Of the 5 infants with transient $\mathrm{HI}$ and a birth weight $Z$ score $>1.2,3$ were associated with maternal gestational diabetes. Rates of cesarean section did not differ significantly between groups, although they were higher in the HI co- hort (61.5\%) than the Canadian population (28.2\%) [16]. Looking at predictors from critical sample biochemistry, there were no significant differences between the 3 groups other than lower cortisol concentration in the infants with transient HI (Table 3).

\section{Hospital Outcome Differences between Groups}

The maximum glucose infusion rate or need for glucagon did not differ between groups (Table 4). However, the DU infants required longer duration of glucagon administration than infants with DR and transient forms of $\mathrm{HI}$ $(p<0.001)$. Feeding issues among all subjects in our HI cohort were extremely common (61.5\%). Infants with the DU subtype were more likely to require a gastrostomy tube for feeding $(p=0.001)$. Length of stay was prolonged 
in the DU group, a trend which persisted with the exclusion of premature infants from the analysis. In a multiregression model controlling for $\mathrm{HI}$ subtype, the presence of feeding problems was associated with an extra 27.7 days in length of stay. Among the entire cohort, birth weight category (SGA vs. appropriate for gestational age vs. LGA) correlated with glucagon duration and length of stay, similar to diagnostic group category, but did not correlate with length of stay when premature infants were excluded.

\section{Discussion}

$\mathrm{HI}$ is an important diagnosis associated with prolonged hospital stays, high rates of feeding problems, and potential serious long-term sequelae. An economic analysis by a group in the UK demonstrated that despite its relative rare occurrence, costs associated with the care of infants with $\mathrm{HI}$ represent a substantial burden [17]. A further study has demonstrated that infants with $\mathrm{HI}$ have significantly longer hospital admissions and costs than gestational age-matched infants with hypoglycemia not secondary to HI [11]. Rapid identification of transient versus persistent forms of $\mathrm{HI}$, and in particular those infants who may have a DU form where genetic testing helps inform management options, is important to reduce the length of admission and optimize management. Algorithms to guide genetic testing once a child is identified as being DU have been published $[4,18]$. We sought to determine key clinical features to aid in differentiating HI subtype, allowing for prompt and targeted management of the disease, even earlier in the disease course.

Birth weight $Z$ score was significantly associated with the subtype of HI. Macrosomia has long been recognized to be associated with the congenital forms of HI secondary to prenatal hypersinulinemia $[1,19]$. In contrast, the transient forms of $\mathrm{HI}$ are commonly seen in infants who are SGA. Our data confirm the incremental increased likelihood of having a persistent and DU form of $\mathrm{HI}$ with increased birth weight. Only 2 infants of the transient HI group (4.3\%) were LGA (birth weight $Z$ score $>1.2$ ) which was not explained by maternal gestational diabetes. Our data are similar to those of a French cohort of 67 infants with transient HI, where only $8 \%$ of the infants had a birth weight $>90$ th percentile, and these data included infants born to mothers with gestational diabetes [20]. Our results strongly suggest that all infants with $\mathrm{HI}$ who are LGA, which is not explained by maternal gestational diabetes, should be considered for expedited genetic testing without first assessing for diazoxide responsiveness.

Clinical Predictors of Transient versus

Persistent Hyperinsulinism
There were no other distinguishing presenting clinical characteristic between the different HI groups. While transient $\mathrm{HI}$ is typically associated with classical risk factors, $26.1 \%$ of this subgroup had no identifiable risk factor, such as SGA, fetal distress, or maternal diabetes. As demonstrated previously, neither the severity of hypoglycemia, as measured either by glucose in the critical sample or glucose infusion requirement, nor the insulin measured at the time of hypoglycemia was predictive for the HI group [21, 22]. Insulin is unstable in circulation and can be secreted episodically; critical samples, even if drawn during an episode of hypoglycemia, are not always reflective of the diagnostic parameters of HI. Cortisol concentrations were more often inappropriately low in the transient group, although the reason for this is unclear. Impaired counter-regulatory hormone response to recurrent hypoglycemia, particularly cortisol, is a wellrecognized phenomenon [23].

HI often leads to prolonged hospitalization, and this was evident in our cohort [11]. The mean length of stay was 48 days, excluding infants born prematurely where factors other than HI may have impacted this number. Our data also clearly demonstrate the clinical outcomes that differ in their severity depending on disease subtype. In particular, infants with DU HI have a prolonged stay in hospital and increased need for nasogastric or gastrostomy tube, highlighting the importance of early targeted management in this patient group. Of note, length of stay was increased in some transient cases as was duration of the glucagon infusion (Table 4). While it is difficult to accurately determine the reasons underlying this, given the retrospective nature of the study, it is likely that additional medical comorbidities (such as need to be nil by mouth and not being able to be commenced on diazoxide) may have impacted these results. There is a high incidence of feeding problems in this group which could contribute to this observation. Furthermore, given the nature of our study being conducted at a tertiary center without a birth unit on site, cases with transient HI would have been unusually difficult to manage presentation to necessitate transfer from a peripheral center. We would expect infants who met the definition of transient $\mathrm{HI}$ but who were managed solely in the peripheral center to have had lower lengths of stay.

The incidence of feeding issues and need for nasogastric feeds while in hospital were high among all groups. Our results are very similar to Banerjee et al. [24], who documented that $75 \%$ of their patients required nasogastric or gastrostomy feeding and had issues with either vomiting or reflux. The etiology behind the increased in-

Horm Res Paediatr 2020;93:297-303 
cidence of feeding problems is unclear, and probable contributing factors include the underlying condition itself and medications used in the management of HI. Glucagon has known gastroesophageal side effects, and prolonged use of this medication along with either fortified feeds or prolonged high glucose infusion rates likely impacts the ability of infants to feed independently, again highlighting the importance of rapid targeted management. Feeding issues have a significant impact on the clinical course of infants with $\mathrm{HI}$ and are an independent contributor to length of hospital stay, even controlling for HI subtype.

This study is a retrospective chart review and is therefore limited by the data already collected at the time of study conceptualization. However, retrospective reviews are well-suited to rare diseases as sample sizes often exceed resource limitations in prospective analyses. This study is also limited by the uncertainty in how best to define HI subgroups, given the lack of a universally agreedupon definition of transient and persistent $\mathrm{HI}$ in the literature, and recognition that some congenital forms of $\mathrm{HI}$ will resolve in a shorter period. Furthermore, not all subjects received genetic testing; the transient group as defined in this study could potentially include transient congenital forms of HI (e.g., HNF4A). The genetic mutation detection rate in our DR and DU groups is, however, very comparable to published rates from large cohorts [25]. The average age of genetic testing reflects practice over the past 5 years in a tertiary referral center. Patients are often initially managed in peripheral centers, which can delay time to genetic testing. Results can take up to 6 weeks, and therefore, it is important to consider clinical factors that could guide management in the interim. Our analysis of biochemical markers of $\mathrm{HI}$ was limited in that it did not include C-peptide, which has shown recent diagnostic promise in $\mathrm{HI}$ but is not routinely ordered at our center [22]. Finally, as all patients were seen in a tertiary referral center, this may have led to a bias in the patient cohort to infants with a more severe presentation of HI. Confirmation of these findings in other clinical settings would help strengthen their generalizability to inform clinical practice recommendations.

\section{Conclusion}

$\mathrm{HI}$ is a severe disease with many potential long-term neurological sequelae, and genetic testing may help inform care in this population. Specifically, early identification of infants with DU HI is essential to help streamline treatment decisions and reduce length of stay. Our data support

that infants who are LGA with HI, not explained by maternal diabetes, should have early genetic testing for the congenital forms of HI. This may help identify a group of infants who need targeted therapy but who are also at increased risk of prolonged length of stay. Other clinical predictors such as insulin concentration at the time of hypoglycemia or the glucose infusion rate required to avoid hypoglycemia are not, in contrast, predictive of HI subtype. Future research is necessary to assess the cost-benefit analyses of early genetic testing and its influence on length of hospitalization and long-term negative sequelae of HI.

\section{Statement of Ethics}

This study was approved by the Research and Ethics Board at the Hospital for Sick Children, Toronto (REB approval No. 1000053997). Approval to waive the need for informed consent was given by the Ethics Committees, given the retrospective nature of the chart review.

\section{Conflict of Interest Statement}

The authors have no conflicts of interest relevant to this article to disclose.

\section{Funding Sources}

No external funding was secured for this study. The authors have no financial relationships relevant to this article to disclose.

\section{Author Contributions}

N.F. was involved in data acquisition and data analysis, and wrote the first draft of the manuscript. J.H. was involved in project design, data analysis, and reviewing the manuscript. Both authors approved the final version of the manuscript.

\section{References}

1 Shah P, Rahman SA, Demirbilek H, Güemes M, Hussain K. Hyperinsulinaemic hypoglycaemia in children and adults. Lancet Diabetes Endocrinol. 2017;5(9):729-42.

2 Burns CM, Rutherford MA, Boardman JP, Cowan FM. Patterns of cerebral injury and neurodevelopmental outcomes after symptomatic neonatal hypoglycemia. Pediatrics. 2008;122(1):65-74.

3 Menni F, de Lonlay P, Sevin C, Touati G, Peigné $\mathrm{C}$, Barbier $\mathrm{V}$, et al. Neurologic outcomes of 90 neonates and infants with persistent hyperinsulinemic hypoglycemia. Pediatrics. 2001;107(3):476-9. 
4 Mohamed Z, Arya VB, Hussain K. Hyperinsulinaemic hypoglycaemia:genetic mechanisms, diagnosis and management. J Clin Res Pediatr Endocrinol. 2012;4(4):169-81.

5 Yorifuji T, Horikawa R, Hasegawa T, Adachi M, Soneda S, Minagawa M, et al. Clinical practice guidelines for congenital hyperinsulinism. Clin Pediatr Endocrinol. 2017;26(3): 127-52.

6 Rahman SA, Nessa A, Hussain K. Molecular mechanisms of congenital hyperinsulinism. J Mol Endocrinol. 2015;54(2):R119-29.

7 Gembal M, Gilon P, Henquin JC. Evidence that glucose can control insulin release independently from its action on ATP-sensitive $\mathrm{K}+$ channels in mouse B cells. J Clin Invest. 1992;89(4):1288-95.

8 Komatsu M, Schermerhorn T, Aizawa T, Sharp GW. Glucose stimulation of insulin release in the absence of extracellular $\mathrm{Ca} 2+$ and in the absence of any increase in intracellular $\mathrm{Ca} 2+$ in rat pancreatic islets. Proc Natl Acad Sci U S A. 1995;92(23):10728-32.

9 Aynsley-Green A, Hussain K, Hall J, Saudubray JM, Nihoul-Fékété C, De Lonlay-Debeney $\mathrm{P}$, et al. Practical management of hyperinsulinism in infancy. Arch Dis Child Fetal Neonatal Ed. 2000;82(2):F98-107.

10 Aynsley-Green A, Dunne MJ, James RF, Lindley KJ. Ions and genes in persistent hyperinsulinaemic hypoglycaemia in infancy: a commentary on the implications for tailoring treatment to disease pathogenesis. J Pediatr Endocrinol Metab. 1998;11(Suppl 1):121-9.
11 Kozen K, Dassios T, Kametas N, Kapoor RR, Greenough A. Transient neonatal hyperinsulinaemic hypoglycaemia: perinatal predictors of length and cost of stay. Eur J Pediatr. 2018; 177(12):1823-9.

12 Fenton TR, Kim JH. A systematic review and meta-analysis to revise the fenton growth chart for preterm infants. BMC Pediatr. 2013; 13:59.

13 Sharma A, Metzger D. Calculator: preterm Zscores, 22-50 weeks.

14 Männistö JME, Maria M, Raivo J, Kuulasmaa T, Otonkoski T, Huopio H, et al. Clinical and genetic characterization of 153 patients with persistent or transient congenital hyperinsulinism. J Clin Endocrinol Metab. 2020;105(4): e1686-94

15 Hoe FM, Thornton PS, Wanner LA, Steinkrauss L, Simmons RA, Stanley CA. Clinical features and insulin regulation in infants with a syndrome of prolonged neonatal hyperinsulinism. J Pediatr. 2006;148(2):20712.

16 Denver R. C-section rates continue to increase while birth rates decline.

17 Eljamel S, Griffiths A, Evans J, Banerjee I, Hussain K, Thompson R. The burden of congenital hyperinsulinism in the United Kingdom: a cost of illness study. Orphanet J Rare Dis. 2018;13(1):123.

18 Banerjee I, Salomon-Estebanez M, Shah P, Nicholson J, Cosgrove KE, Dunne MJ. Therapies and outcomes of congenital hyperinsulinism-induced hypoglycaemia. Diabet Med. 2019;36(1):9-21.
19 Stanley CA, Baker L. Hyperinsulinism in infants and children: diagnosis and therapy. Adv Pediatr. 1976;23:315-55.

20 Louvigne M, Rouleau S, Caldagues E, Souto I, Montcho Y, Bouvagnet AM, et al. Association of maternal nutrition with transient neonatal hyperinsulinism. PLoS One. 2018;13(5): e0195383.

21 Al-Otaibi H, Senniappan S, Alam S, Hussain $\mathrm{K}$. Biochemical studies in patients with hyperinsulinaemic hypoglycaemia. Eur J Pediatr. 2013;172(11):1435-40.

22 Ferrara C, Patel P, Becker S, Stanley CA, Kelly A. Biomarkers of Insulin for the diagnosis of hyperinsulinemic hypoglycemia in infants and children. Eur J Pediatr. 2016;168:212-9.

23 Hussain K, Hindmarsh P, Aynsley-Green A. Neonates with symptomatic hyperinsulinemic hypoglycemia generate inappropriately low serum cortisol counterregulatory hormonal responses. J Clin Endocrinol Metab. 2003;88(9):4342-7.

24 Banerjee I, Forsythe L, Skae M, Avatapalle HB, Rigby L, Bowden LE, et al. Feeding problems are persistent in children with severe congenital hyperinsulinism. Front Endocrinol. 2016;7:8

25 Snider KE, Becker S, Boyajian L, Shyng SL, MacMullen C, Hughes N, et al. Genotype and phenotype correlations in 417 children with congenital hyperinsulinism. J Clin Endocrinol Metab. 2013;98(2):e355-63. 